\title{
Resonator stability and higher-order modes in free-electron laser oscillators
}

\author{
Abhishek Pathak $^{1}$ and Srinivas Krishnagopal ${ }^{1,2}$ \\ ${ }^{1}$ Bhabha Atomic Research Centre, Trombay, Mumbai 400 085, India \\ ${ }^{2}$ Centre for Excellence in Basic Sciences, University of Mumbai, Mumbai 400 098, India
}

(Received 7 March 2014; published 7 August 2014)

\begin{abstract}
Three-dimensional simulation codes GENESIS and OPC are used to investigate the dependence of the resonator stability of free-electron laser (FEL) oscillators on the stability parameter, laser wavelength, outcoupling hole size and mirror tilt. We find that to have stable lasing over a wide range of wavelengths, the FEL cavity configuration should be carefully chosen. Broadly, the concentric configuration gives nearGaussian modes and the best performance. At intermediate configurations the dominant mode often switches to a higher-order mode, which kills lasing. For the same reason, the outcoupled power can also be less. We have constructed a simple analytic model to study resonator stability which gives results that are in excellent agreement with the simulations. This suggests that modes in FEL oscillators are determined more by the cavity configuration and radiation propagation than by the details of the FEL interaction. We find (as in experiments at the CLIO FEL) that tilting the mirror can, for some configurations, lead to more outcoupled power than a perfectly aligned mirror because the mode is now a more compact higher-order mode, which may have implications for the mode quality for user experiments. Finally, we show that the higher-order mode obtained is usually a single Gauss-Laguerre mode, and therefore it should be possible to filter out the mode using suitable intracavity elements, leading to better FEL performance.
\end{abstract}

DOI: 10.1103/PhysRevSTAB.17.080703

PACS numbers: 41.60.Cr, 42.60.Da, 52.59.Rz

\section{INTRODUCTION}

Most infrared free-electron laser (FEL) facilities around the world use thermionic electron sources with modest currents $(\approx 10-100$ A) [1-5] and therefore operate the FEL in the oscillator configuration, very often with on-axis hole outcoupling. The optical resonator plays an important role in determining the dominant mode within the cavity. The mode itself determines the overlap between the electron beam and the radiation, affecting the FEL gain and hence lasing. In addition, the shape of the dominant mode on the outcoupling mirror also determines the outcoupled power.

It is well known [6-8] that mode competition and mode beating affect the stability of optical modes in FEL oscillators and therefore constrain the operating parameters of the FEL. This can be a concern for FEL facilities, because one of the important advantages of FELs is the ability to provide tunable coherent radiation over a large wavelength range. For this to be possible, one has to ensure that the mode is stable over the entire range. Another important issue for an FEL facility is to ensure substantial outcoupled power to the user. Maximum power will be outcoupled when the mode profile on the outcoupling mirror is Gaussian, and therefore maximum intensity is intercepted by the hole. However, if the beam is

Published by the American Physical Society under the terms of the Creative Commons Attribution 3.0 License. Further distribution of this work must maintain attribution to the author $(s)$ and the published article's title, journal citation, and DOI.
non-Gaussian with a minimum at the center, as may be the case with some higher-order modes, then the outcoupled power will be small, even if there is lasing. Consequently, a detailed understanding of stability in FEL oscillators involves a study of the resonator modes, which in turn depend in a complicated way on a number of essential parameters, such as the radiation wavelength, resonator configuration, hole size, electron beam current, mirror tilt etc. Therefore, it is typically necessary to take recourse to three-dimensional FEL codes such as GINGER [9] or GENESIS [10].

Here we present a detailed study of transverse optical modes in FEL oscillators as a function of different cavity and radiation parameters. We find that the nature of the dominant mode can change quite dramatically as one or more of these parameters are varied.

We also propose a simple matrix-based model for studying the evolution of the mode. Our model includes the FEL interaction as a thin lens located at the center of the undulator. While this model certainly does not include the details of the FEL interaction, we show that it goes a long way in explaining the modes in the FEL oscillator, and agrees well with the three-dimensional simulations. This shows that the modes in the FEL oscillator are determined more by the cavity configuration and radiation propagation than by the details of the FEL interaction.

Modes in cavities can always be expanded in terms of a complete set of Gauss-Laguerre (GL) or Gauss-Hermite (GH) modes [11-13]; for an axisymmetric resonator the former are a more natural choice. We show that most of the 
non-Gaussian modes obtained are single higher-order GL modes. This identification suggests that the use of intracavity elements for suppressing the higher-order modes can lead to better operation of the FEL, in terms of greater outcoupled power and operation over a wider range of wavelengths.

\section{MATRIX MODEL}

In terms of a complex parameter $q$, a two-dimensional Gaussian beam can be written as [11]

$$
G(x, y, z)=\frac{1}{q(z)} e^{-i k \frac{r^{2}}{2 q(z)},}
$$

where

$$
\frac{1}{q(z)}=\frac{1}{R(z)}-i \frac{\lambda}{\pi w^{2}(z)}
$$

Here $k$ is the wave vector, $\lambda$ is the wavelength, $R(z)$ is the radius of curvature of the wave front and $w(z)$ is the beam spot size. We have assumed that the beam is circularly symmetric, so that $q_{x}=q_{y}=q$ and $x^{2}+y^{2}=r^{2}$. This beam can be propagated through an optical system given its transfer matrix using the equation

$$
\left[\begin{array}{c}
q_{2} \\
1
\end{array}\right]=\left[\begin{array}{ll}
A & B \\
D & C
\end{array}\right]\left[\begin{array}{c}
q_{1} \\
1
\end{array}\right]
$$

where A, B, C, D are determined by the transfer operation performed on the beam. A generalized operation of such a ray matrix on the given $2 \mathrm{D}$ beam leads to the transformation of the $q$ parameter as follows:

$$
q_{2}=\frac{A q_{1}+B}{C q_{1}+D} .
$$

This, in combination with a knowledge of the Guoy phase shift for the beam, allows us to analytically study the evolution of the transverse mode of the beam as it propagates inside a resonator. For a cold, closed cavity, the transformations are just (two) drifts and (two) reflections, for which the matrix elements are well known [11]. Using these matrices, a simple analytic model has been implemented in MATLAB [14]. We use this model to study the evolution of the transverse mode profile; we have not considered the longitudinal dimension, since our focus here is on transverse modes.

Once the FEL interaction is introduced in the resonator the stability diagram will be modified. The degree of modification will depend on the strength of the interaction, and therefore on the electron beam current. As the current increases, the strength of the interaction increases, resulting in well-known phenomena such as gain guiding and refractive guiding [15]. Therefore, it is natural to expect that, to first order, the FEL interaction acts as a lens, to focus the optical beam.

In our analytic model, therefore, we chose to model the FEL interaction as a thin lens located at the center of the resonator. The matrix for a thin lens is also well known [11]. For the focal length of the lens, we used the data from the simulations, and calculated an effective focal length by taking the actual size of the optical beam at the downstream mirror, and using the standard formula for the focal length assuming a Gaussian optical beam [11],

$$
f=\frac{\pi w(z) w_{0}}{\lambda},
$$

where $w_{0}$ is the waist size. Note that this value of the focal length is a function of current. As the current increases, the spot size on the downstream mirror decreases, and so does the focal length. However, this calculated value of the focal length is always much greater than the length of the resonator.

\section{CLOSED CAVITY (NO OUTCOUPLING HOLE)}

We start with a study of a closed cavity. In the first subsection we review the case for a cold closed cavity, i.e. when there is no FEL interaction, for which analytic results are well known. While this is of course unrealistic, it does substantially simplify the cavity dynamics, and serves as a benchmark for our simple model. We then consider the case when there is an FEL interaction.

\section{A. Cold-cavity case}

Consider an empty optical resonator consisting of two concave mirrors (with no holes), of radii of curvature $R_{1}$ and $R_{2}$, placed a distance $L$ apart. In terms of the stability parameters, $g_{1,2}=\left(1-L / R_{1,2}\right)$, one can show from a simple matrix analysis, using geometrical optics, that the stability criterion is $0 \leq g_{1} g_{2} \leq 1$. Further, if we consider a symmetrical resonator, so that $R_{1}=R_{2}$, then $g_{1}=g_{2}(=g$, say), and the stability criterion reduces to $-1 \leq g \leq 1$.

As a test case, we decided to study this simple system using full-blown FEL simulations, as well as with our analytic model. We allowed for finite reflectivity of the mirrors. Note that the cavity mode will be the lowest order Gaussian $\left(T E M_{00}\right)$ only when there is no significant power loss in the cavity.

For the simulations we used the FEL code GENESIS, and for propagating the radiation from the exit of the undulator, through the optical cavity, back to the entrance of the undulator, optical propagation code (OPC) was used [16]. We modeled the empty resonator by making the current zero in the FEL simulations. For the matrix analysis as well as the GENESIS+OPC simulations, we kept the radius of curvature of the mirrors fixed, $R_{1}=R_{2}=6.15 \mathrm{~m}$, varied their separation in order to vary the stability parameter $g$, 
TABLE I. Parameters used in the simulations.

\begin{tabular}{lc}
\hline \hline Parameter & Value \\
\hline Initial power & $1 \mathrm{MW}$ \\
Wavelength & $10-60 \mu \mathrm{m}$ \\
Radius of curvature of mirrors & $6.15 \mathrm{~m}$ \\
Mirror size & $23 \mathrm{~mm}$ \\
Reflectivity of the mirrors & 0.99 \\
Cavity length & $6.15-12.4 \mathrm{~m}$ \\
Hole radius & $1-4 \mathrm{~mm}$ \\
Outcoupled mirror tilt & $0-5 \mathrm{mrad}$ \\
Undulator type & Helical \\
Undulator length & $2 \mathrm{~m}$ \\
Undulator period & $0.05 \mathrm{~m}$ \\
Undulator parameter & 0.637 \\
\hline \hline
\end{tabular}

and looked at the nature of the dominant mode. The simulation parameters are shown in Table I.

For a mirror reflectivity of unity, i.e. when there is no loss, we always find the dominant mode to be Gaussian, in the range $-1 \leq g \leq 0$, as expected. For a mirror reflectivity of 0.99 too, we find that the mode is Gaussian, even though there is loss. This is because there is no radial dependence to the loss, and therefore there can be no change in the mode; the mode can change only if there is a radial dependence to the reflectivity, such as due to loss at the edges of the mirror. These results are shown for the simulations as well as our analytic model in Fig. 1. Note that here, and everywhere else in the paper, modes are shown when the optical beam falls on the outcoupling mirror. These results serve to benchmark our model.

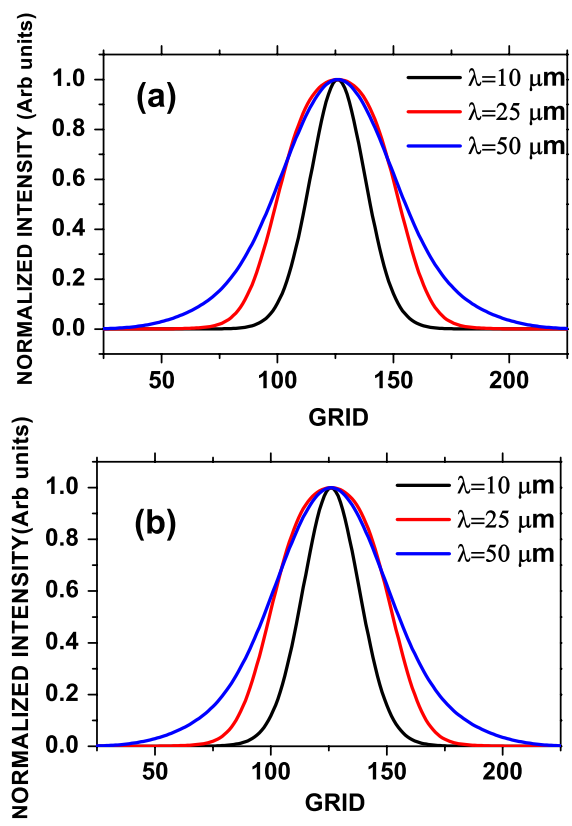

FIG. 1. For a cold, closed cavity in the confocal configuration, cross section of the transverse mode at the downstream mirror using (a) GENESIS+OPC, and (b) our analytic model.

\section{B. FEL interaction}

Next, we introduce the FEL interaction in the simulations and in our model. In the confocal configuration, at wavelengths of 10,25 , and $50 \mu \mathrm{m}$, the optical beam is focused to a tighter spot as the current is increased, with no major change in the mode. This effect is seen for the simulations in Fig. 2, which shows a lineout of the laser pulse profile for different currents. It can be seen that as the current increases the pulse becomes sharper. In the concentric configuration, at the same wavelengths, we found very little focusing of the mode, for the same range of beam currents. For both configurations our analytic model gives the same results.

Figure 3 shows a plot of the spot size as a function of current, at a wavelength of $10 \mu \mathrm{m}$ for both concentric as

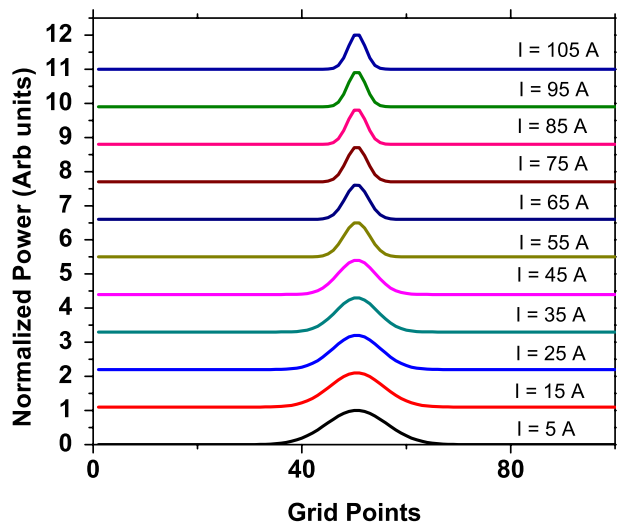

FIG. 2. For a closed cavity in the confocal configuration and a wavelength of $10 \mu \mathrm{m}$, with an FEL interaction, cross section of the transverse mode as a function of electron current, obtained from the simulations.

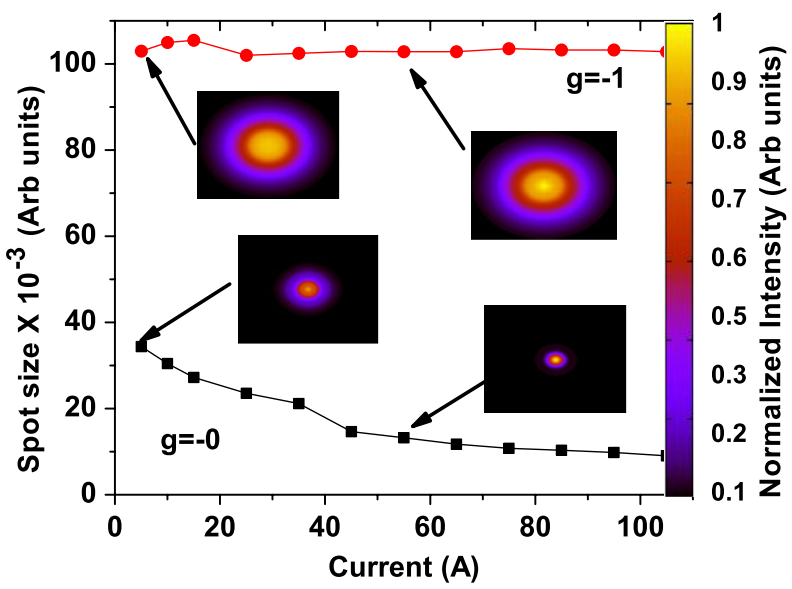

FIG. 3. FWHM of the transverse modes obtained in the simulations, at a wavelength of $10 \mu \mathrm{m}$, as a function of electron current for the confocal $(g=0)$ and concentric $(g=-1)$ configurations. Plots of the modes at 5 and $55 \mathrm{~A}$, for the confocal and concentric configurations, are also shown. For each plot the window size is $50 \mathrm{~mm}$ by $50 \mathrm{~mm}$. 

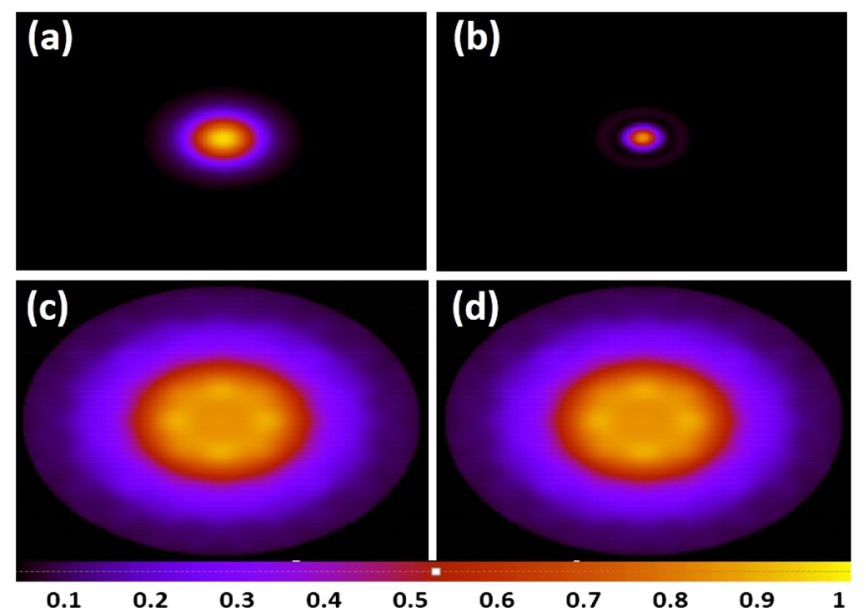

Normalized Intensity (Arb units)

FIG. 4. Mode profiles obtained from our analytic model, at a wavelength of $10 \mu \mathrm{m}$, in the confocal configuration with an electron beam current of (a) $I=5 \mathrm{~A}$ and (b) $I=55 \mathrm{~A}$, and in the concentric configuration with (c) $I=5 \mathrm{~A}$ and (d) $I=55$ A. For each plot the window size is $50 \mathrm{~mm}$ by $50 \mathrm{~mm}$.

well as confocal configurations, from the simulations, which shows these features very clearly. Also shown are the mode profiles from the simulations, at a few currents. The focusing of the spot for the confocal configuration, and the lack of focusing for the concentric, are clearly seen. Figure 4 shows the modes from our analytic model, for the concentric and confocal configurations, each at two different currents. The same features are clearly seen from these plots too.

\section{CAVITY WITH HOLE OUTCOUPLING}

The more realistic case is, of course, when there is a hole in one of the mirrors, so that radiation can be outcoupled. However, with the introduction of an on-axis hole, there is clearly going to be a substantial perturbation to the equilibrium mode and hence the FEL performance $[12,17,18]$. Here we introduce an outcoupling hole on the downstream mirror and study the situation systematically, as a function of cavity length (i.e. stability parameter), wavelength and hole size. Parameters used in the simulations are shown in Table I.

\section{A. Dependence on cavity length}

To study how the dominant or equilibrium mode varies with the stability parameter $g(=1-L / R)$, we varied the length $L$ of the resonator while keeping the radius of curvature $R$ of the mirrors fixed. Earlier we have studied this for a closed cavity, with no outcoupling hole. Here we study the stability for a resonator with an outcoupling hole, using GENESIS+OPC simulations for different cavity lengths, at three different FEL wavelengths and for four different sizes of the outcoupling hole.
Here we also looked at the transverse modes at the points where the outcoupled power decreases and also where there is no lasing. In our simulations, the optical radiation is initialized at the entrance to the undulator, the undulator is symmetric within the resonator and the Rayleigh length of the optical beam is around half of the undulator length. The radiation will subsequently propagate self-consistently, and its shape will evolve. In particular, when it evolves into a higher-order mode, naturally the peak intensity will be away from the center, where the electron beam is, and therefore there will be a reduction in the overlap and hence the filling factor. This is precisely why lasing can be killed.

Figure 5 shows the outcoupled power as a function of the stability parameter $(g)$, at three different wavelengths, and two different hole sizes. Figure 5(a) shows that for a hole size of $1 \mathrm{~mm}$ lasing is seen for all configurations between the confocal $(g=0)$ and the concentric $(g=-1)$. At the longest wavelength of $50 \mu \mathrm{m}$ there is little change in the outcoupled power with $g$. However, at $10 \mu \mathrm{m}$ there is an intermediate range of cavity lengths, corresponding to $-0.8 \leq g \leq-0.2$, where there is a large fluctuation in the power, suggesting instability in the mode. The situation is worse with a $4 \mathrm{~mm}$ hole, Fig. 5(b). At a wavelength of $10 \mu \mathrm{m}$, there is no lasing for $-0.95 \leq g \leq-0.80$, and again for $-0.65 \leq g \leq-0.20$. Even at a longer wavelength of $25 \mu \mathrm{m}$, there is no lasing for $-0.65 \leq g \leq-0.30$. Only the longest wavelength of $50 \mu \mathrm{m}$ shows lasing over the entire range.
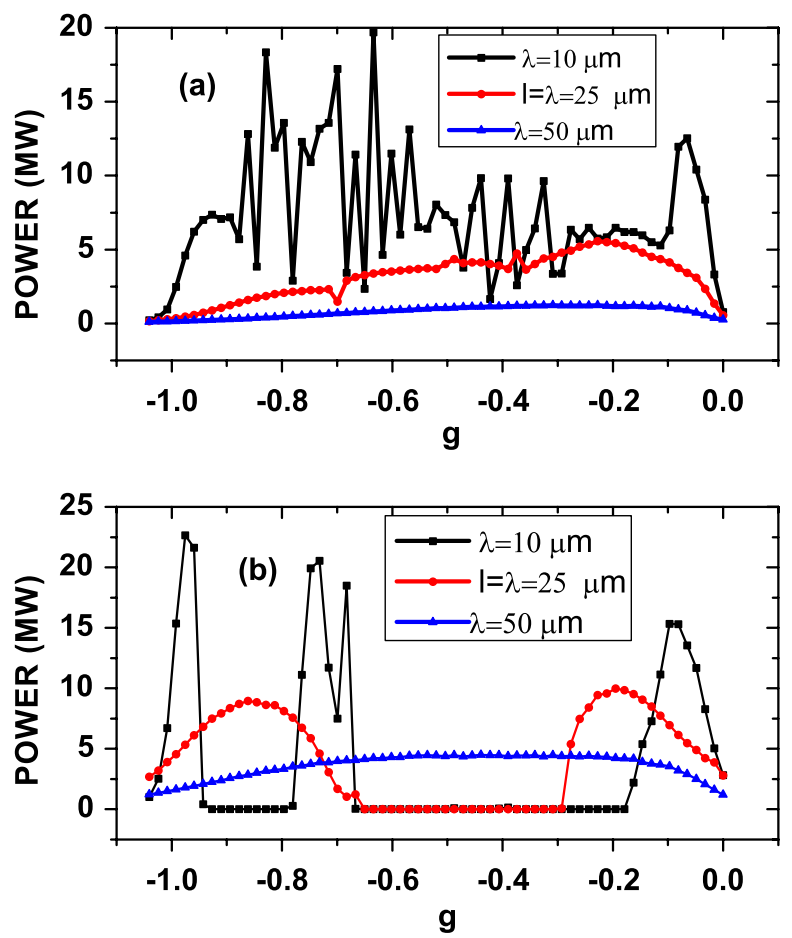

FIG. 5. Variation of outcoupled power as a function of the stability parameter $(g)$, for different wavelengths, with a hole size of (a) $1 \mathrm{~mm}$ and (b) $4 \mathrm{~mm}$. 


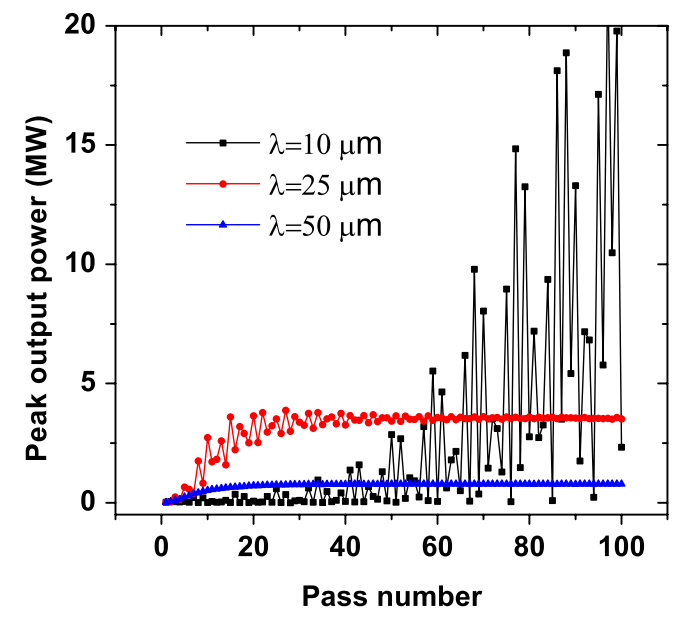

FIG. 6. Outcoupled power as a function of pass number, for three different wavelengths, with a hole size $1 \mathrm{~mm}$, and $g=-0.6$.

The lack of stability observed in Fig. 5(a) can be seen more clearly in a plot of the outcoupled power as a function of pass number, Fig. 6. At a wavelength of $50 \mu \mathrm{m}$, with a $1 \mathrm{~mm}$ hole, one can see that the power rises and saturates smoothly. At $25 \mu \mathrm{m}$, the power initially fluctuates a little, but finally saturates. But, at a wavelength of $10 \mu \mathrm{m}$ the pass-to-pass fluctuations in the outcoupled power are large, and keep increasing with time, indicative of mode instability, as a consequence of which there is no lasing. For the larger hole size of $4 \mathrm{~mm}$, no lasing was observed at the shorter wavelengths ( 10 and $25 \mu \mathrm{m}$ ), but at $50 \mu \mathrm{m}$ there is lasing and the power saturates smoothly, indicating that there is no mode beating.

Figure 7 shows the mode profiles for the confocal, concentric and an intermediate $(g=-0.6)$ configuration, for a wavelength of $25 \mu \mathrm{m}$ and a $1 \mathrm{~mm}$ hole. It can be seen that for the confocal configuration the mode is substantially non-Gaussian, with a dip at the center, while for the concentric configuration the mode, though slightly larger, is more Gaussian-like, with a maximum at the center. For the intermediate configuration the mode is an excellent Gaussian. This explains why, for the wavelength of $25 \mu \mathrm{m}$

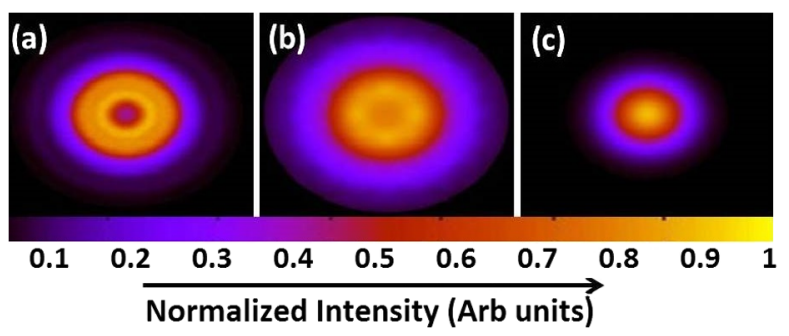

FIG. 7. Mode profiles from the simulations at a wavelength of $25 \mu \mathrm{m}$ and a hole size of $1 \mathrm{~mm}$, for the (a) confocal $(g=0)$, (b) concentric $(g=-1)$ and (c) intermediate $(g=-0.6)$ configurations. For each plot the window size is $50 \mathrm{~mm}$ by $50 \mathrm{~mm}$. and hole size of $1 \mathrm{~mm}$, the power is maximum for $g=-0.6$, and is less for the confocal and concentric configurations.

\section{B. Wavelength dependence}

From the experimental point of view, since the resonator geometry cannot be changed, it is more relevant to fix the cavity length, i.e. value of $g$, and look at the outcoupled power as a function of wavelength, for different hole sizes.

Figure 8 shows the outcoupled power as a function of wavelength for three different resonator configurations: confocal $(g=0)$, concentric $(g=-1)$, and an intermediate configuration with $g=-0.6$. Figure 8(a) shows that with a smaller, $1 \mathrm{~mm}$, hole essentially all wavelengths lase, though $g=-0.6$ gives the best performance, in terms of maximum outcoupled power. The concentric and confocal resonators behave similarly, and the outcoupled power decreases with increasing wavelength, because of the greater diffraction. For a $4 \mathrm{~mm}$ hole, Fig. 8(b) shows that the concentric and confocal configurations lase at all wavelengths, with outcoupled power decreasing with increasing wavelength, as was the case with a $1 \mathrm{~mm}$ hole. For $g=-0.6$ lasing commences only after around $30 \mu \mathrm{m}$. Thus, for the concentric and confocal configurations the overall behavior is similar for both hole sizes, except for the fact that with a larger hole one can couple out more power. However, the $g=-0.6$ configuration is more sensitive to the hole size.
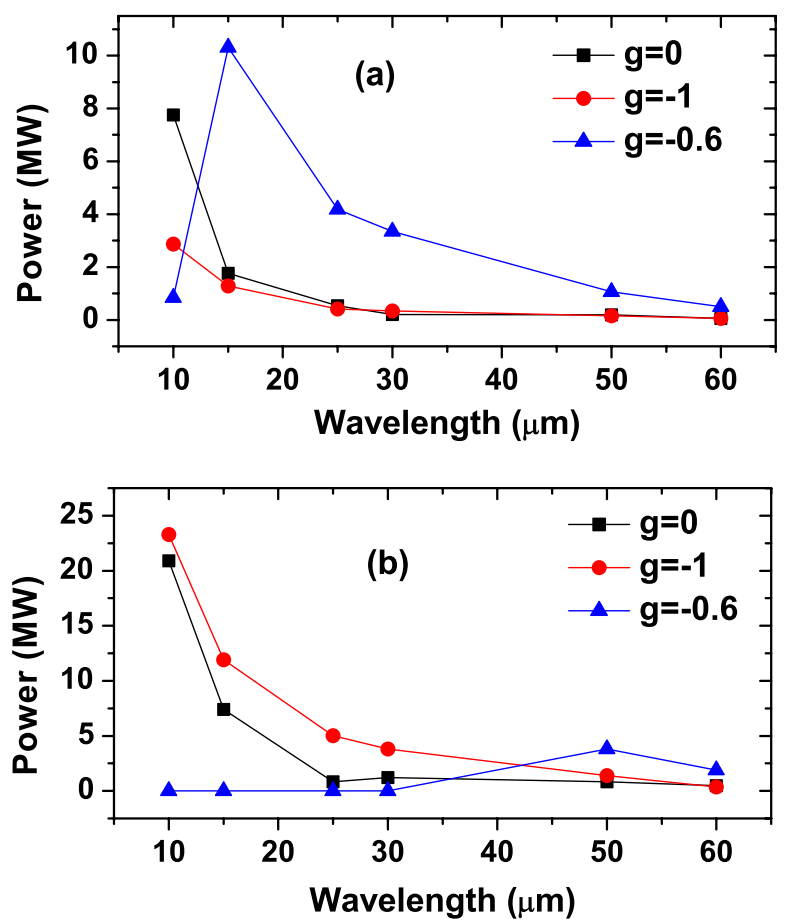

FIG. 8. Variation of outcoupled power as a function of wavelength for the confocal $(g=0)$, concentric $(g=-1)$, and intermediate $(g=-0.6)$ configurations. For a hole size of (a) $1 \mathrm{~mm}$ and (b) $4 \mathrm{~mm}$. 


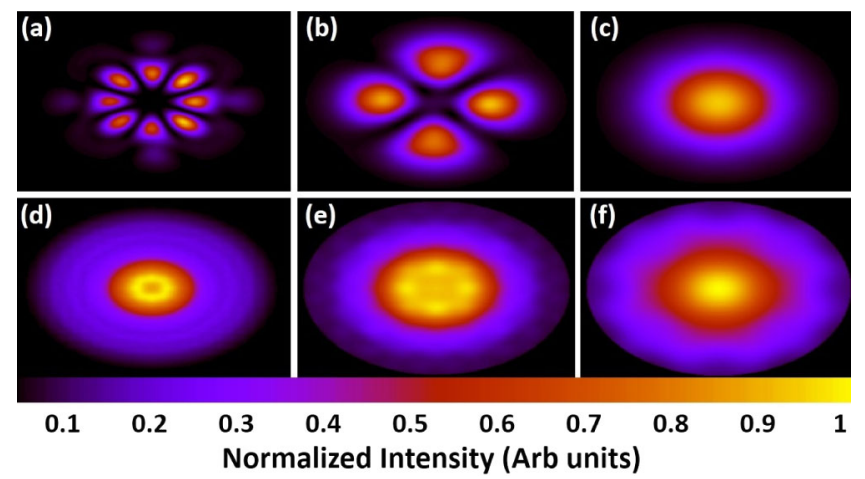

FIG. 9. Mode profiles from the simulations with an outcoupling hole size of $4 \mathrm{~mm}$, for the intermediate configuration $(g=-0.6)$ at wavelengths of (a) $10 \mu \mathrm{m}$, (b) $25 \mu \mathrm{m}$ and (c) $50 \mu \mathrm{m}$, and for the concentric configuration $(g=-1)$ at wavelengths of (d) $10 \mu \mathrm{m}$, (e) $25 \mu \mathrm{m}$ and (f) $50 \mu \mathrm{m}$. For each plot the window size is $50 \mathrm{~mm}$ by $50 \mathrm{~mm}$.

These are important observations. They suggest that the FEL cavity configuration should be chosen carefully, in order to have stable lasing over a wide range of wavelengths, and concentric or confocal configurations may be preferable. For FELs with an intermediate value of $g$, such as the CLIO FEL in France [19], there is a danger that it may not be possible to lase at shorter wavelengths, especially if the hole size is large.

In order to better understand the observed behavior, we looked at the modes on the outcoupling mirror, corresponding to some of the points in Fig. 8. For a hole size of $4 \mathrm{~mm}$ and wavelengths of 10,25 , and $50 \mu \mathrm{m}$, we looked at the modes for $g=-1$ and $g=-0.6$, shown in Fig. 9. It can be seen from Figs. 9(a)-9(c) that at $g=-0.6$ and for wavelengths of 10 and $25 \mu \mathrm{m}$ the dominant mode is a higherorder mode with no intensity at the center, and therefore there is no lasing. At $50 \mu \mathrm{m}$ the mode is Gaussian, and therefore there is lasing and a fair amount of outcoupled power. For the concentric configuration, Figs. 9(d)-9(f) show that the modes are essentially Gaussian, and this explains the large outcoupled power in Fig. 8(b). The mode is more compact at shorter wavelengths, which explains why there is more outcoupled power.

For the six cases shown in Fig. 9, we ran our simple analytic model with a hole in the outcoupling mirror. Of course, with the FEL interaction present, this simple model, which has no gain in it, is not expected to give dependable values for the outcoupled power. However, we performed these studies to look at the nature of the transverse mode. These results are shown in Fig. 10. Comparing Figs. 9 and 10 one can see that the agreement is remarkable; our model does an excellent job of reproducing the modes, even though the FEL model is overly simplified. This is because the equilibrium mode in the cavity is determined primarily by the resonator dynamics, to which the FEL interaction acts only as a perturbation.
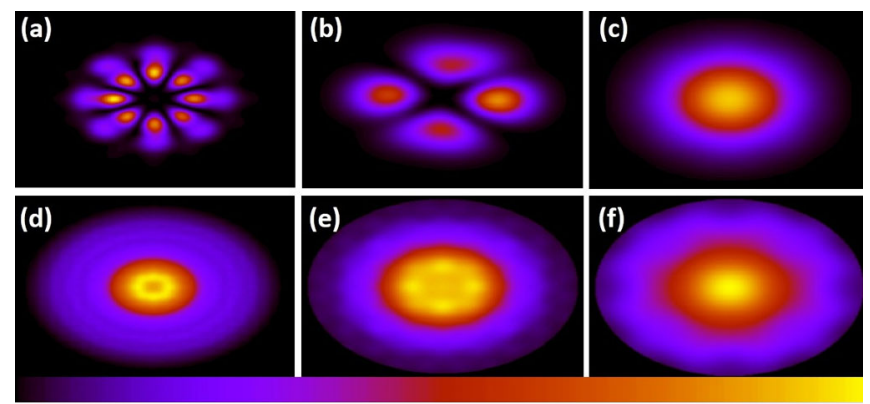

0.1

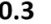

Normalized Intensity (Arb units)

FIG. 10. Mode profiles from our analytic model with an outcoupling hole size of $4 \mathrm{~mm}$, for the intermediate configuration ( $g=-0.6$ ) at wavelengths of (a) $10 \mu \mathrm{m}$, (b) $25 \mu \mathrm{m}$ and (c) $50 \mu \mathrm{m}$, and for the concentric configuration $(g=-1)$ at wavelengths of (d) $10 \mu \mathrm{m}$, (e) $25 \mu \mathrm{m}$ and (f) $50 \mu \mathrm{m}$. For each plot the window size is $50 \mathrm{~mm}$ by $50 \mathrm{~mm}$.

This is an important observation. As we have argued above, and seen in the GENESIS+OPC simulations, for a given resonator configuration the mode can be very different for different wavelengths. In particular, some wavelengths may simply not lase because the mode is non-Gaussian. Therefore, it is important to understand the details of the resonator modes, and these results suggest that it is possible to do this study with our simple analytic model, and without the need for detailed three-dimensional FEL simulations. These resonator studies can then serve as inputs for the detailed FEL design, in order to ensure lasing over the full range of wavelengths for which the FEL facility is being designed.

\section{Dependence on hole size}

We also looked at the hole-size dependence of the outcoupled power, for three different wavelengths, for the confocal $(g=0)$ concentric $(g=-1)$ and intermediate $(g=-0.6)$ configurations.

Figures 11(a) and 11(b) show that for the confocal and concentric configurations, the outcoupled power increases with increasing hole size. This shows that increasing the hole size does not substantially alter the mode profile, and only allows for more power to be coupled out. For the intermediate case, however, the situation is different. Figure 11(c) shows that at the shortest wavelength, $10 \mu \mathrm{m}$, there is no lasing after a hole size of $2 \mathrm{~mm}$. At $25 \mu \mathrm{m}$, the outcoupled power initially increases with hole size, but then starts falling. At $50 \mu \mathrm{m}$, there is a monotonic rise in the outcoupled power, but the increase is slower after a hole size of $3 \mathrm{~mm}$. All these features can be attributed to a change in the transverse mode profile.

For the confocal configuration $(g=0)$, Fig. 11(a), for a wavelength of $10 \mu \mathrm{m}$, we find that the modes are nonGaussian, with a dip at the center, for all four hole sizes; the mode profile for the $1 \mathrm{~mm}$ hole is shown in Fig. 12(a). As a 

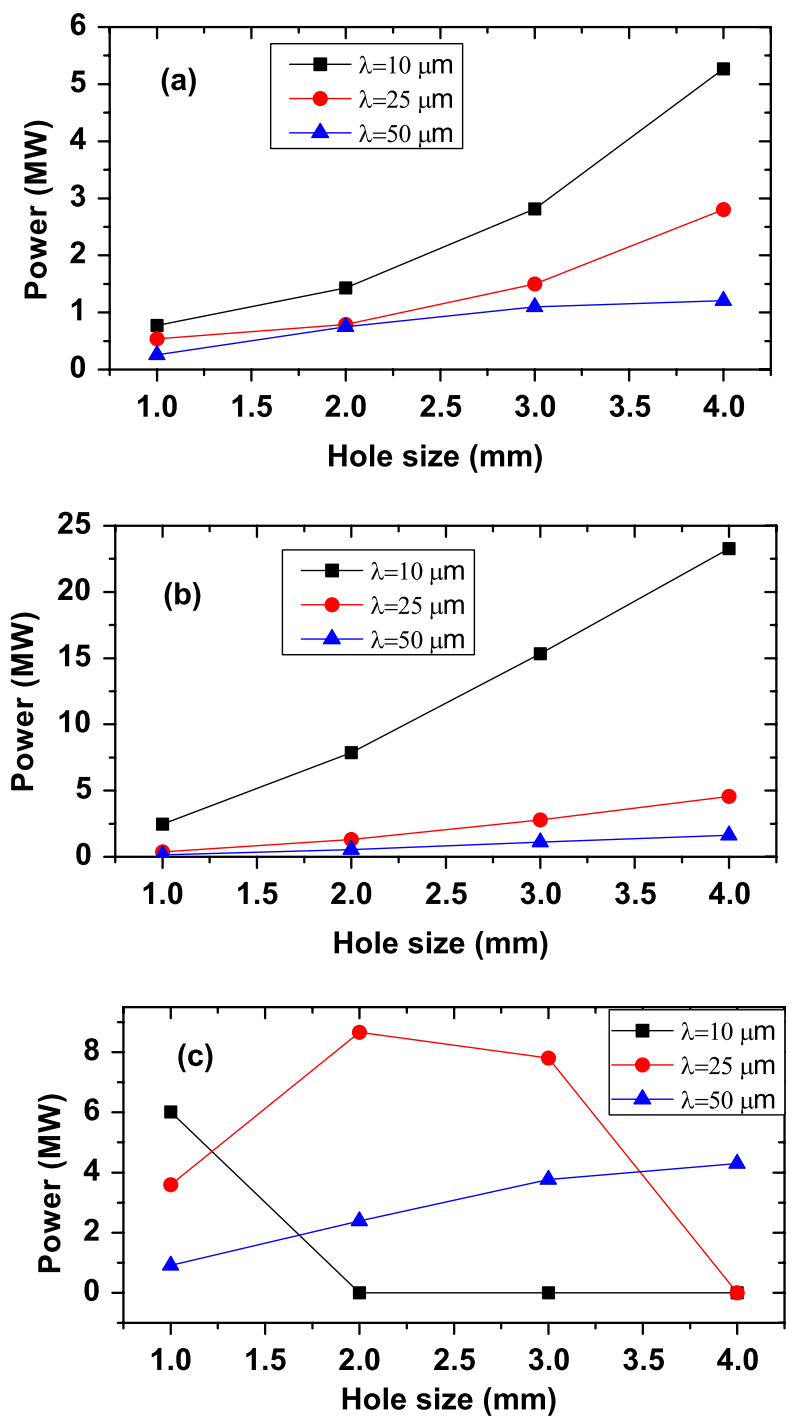

FIG. 11. Outcoupled power as a function of hole size at three different wavelengths for the (a) confocal $(g=0)$, (b) concentric $(g=-1)$ and (c) intermediate $(g=-0.6)$ configurations.

consequence, for smaller hole sizes less power is coupled out. As the hole size increases, it intercepts the maximum of the mode, and therefore the outcoupled power increases substantially, as shown in Fig. 11(a). At $25 \mu \mathrm{m}$ too, the mode has a dip at the center [the mode profile for a $1 \mathrm{~mm}$ hole is shown in Fig. 7(a)], and therefore the same dependence on hole size is seen. At $50 \mu \mathrm{m}$ the modes are Gaussian at all hole sizes, and so the power increases only very gradually with increasing hole size.

For the concentric configuration $(g=-1)$, Fig. 11(b), at all wavelengths and all hole sizes, the mode is Gaussian or close to Gaussian (only a small dip at the center). Therefore, for a given wavelength the outcoupled power increases with hole size, as more and more of the beam is intercepted, and as the wavelength increases the outcoupled power drops, because of the greater diffraction. The modes for the $4 \mathrm{~mm}$ hole size, for wavelengths of 10,25 , and

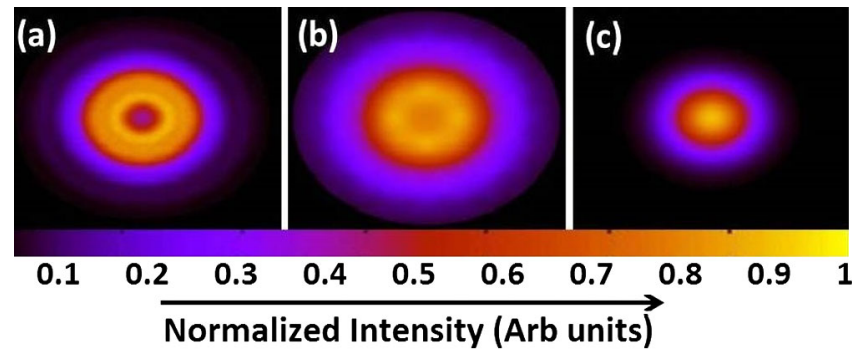

FIG. 12. Mode profiles from the simulations at a wavelength of $10 \mu \mathrm{m}$, for a hole size of $1 \mathrm{~mm}$, for the (a) confocal $(g=0)$, (b) concentric $(g=-1)$ and (c) intermediate $(g=-0.6)$ configurations. For each plot the window size is $50 \mathrm{~mm}$ by $50 \mathrm{~mm}$.

$50 \mu \mathrm{m}$, are shown in Figs. 9(d)-9(f). The mode profile for a wavelength of $10 \mu \mathrm{m}$ and $1 \mathrm{~mm}$ hole size is shown in Fig. 12(b). They are all seen to be Gaussian or close to Gaussian.

For the intermediate configuration $(g=-0.6)$, Fig. 11(c), the situation is more interesting. At a wavelength of $10 \mu \mathrm{m}$, substantial power is coupled out only for a hole size of $1 \mathrm{~mm}$; at all other hole sizes there is no lasing. This is because for the $1 \mathrm{~mm}$ hole size, the mode is near Gaussian, with a maximum at the center, Fig. 12(c). For all other hole sizes, the dominant mode is a higher-order mode with no field at the center, and therefore there is no lasing. For the $4 \mathrm{~mm}$ hole, the mode is shown in Fig. 9(a), where it can been seen that the mode is completely distorted from Gaussian. At a wavelength of $25 \mu \mathrm{m}$, the modes at the first three hole sizes are roughly Gaussian, and therefore there is lasing with substantial outcoupled power. For a $4 \mathrm{~mm}$ hole, however, the mode is a higher-order mode with a minimum at the center, as shown in Fig. 9(b), and therefore there is no lasing. Finally, at a wavelength of $50 \mu \mathrm{m}$, the mode is Gaussian at all hole sizes, and therefore the outcoupled power simply increases with hole size; the mode for a $4 \mathrm{~mm}$ hole is shown in Fig. 9(c), where the Gaussian nature is evident.

The results of this section show that the intermediate configuration is very sensitive to the hole size, while the confocal and concentric are much less sensitive. This can be explained as a consequence of a change of the dominant mode with hole size in the former case, which does not happen in the latter two cases.

\section{ROLE OF MIRROR TILT}

In FEL simulations, the two mirrors of the resonator are assumed to be parallel to each other. Of course, in reality this can never be exactly true. It is therefore important to ask how much tilt can be tolerated, without changing the optical mode in the resonator. In order to study this effect, we took advantage of the features available in the OPC code, which allows such tilts in the mirror. We investigated the behavior of the mode for all three resonator configurations and all three wavelengths that we have studied above, for a hole size of $4 \mathrm{~mm}$. For simplicity the tilt was introduced 
uniformly in both $x$ and $y$ directions. We could not incorporate this feature in our analytic model.

Figure 13 shows the output power as a function of tilt on the outcoupling mirror for all three resonator

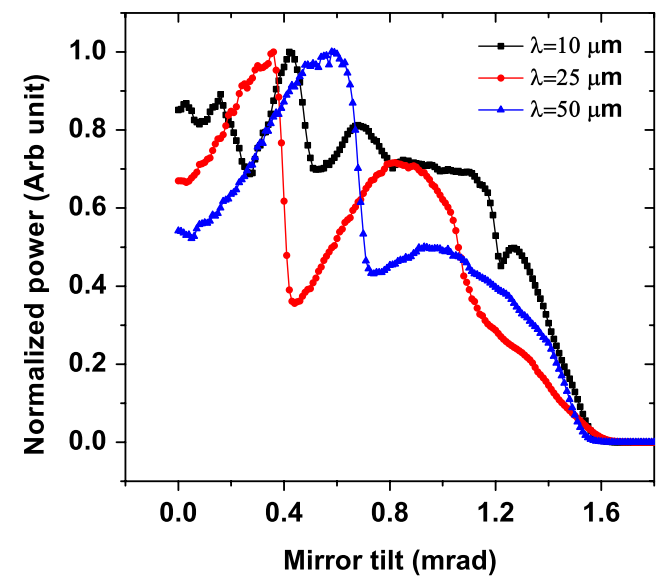

(a)

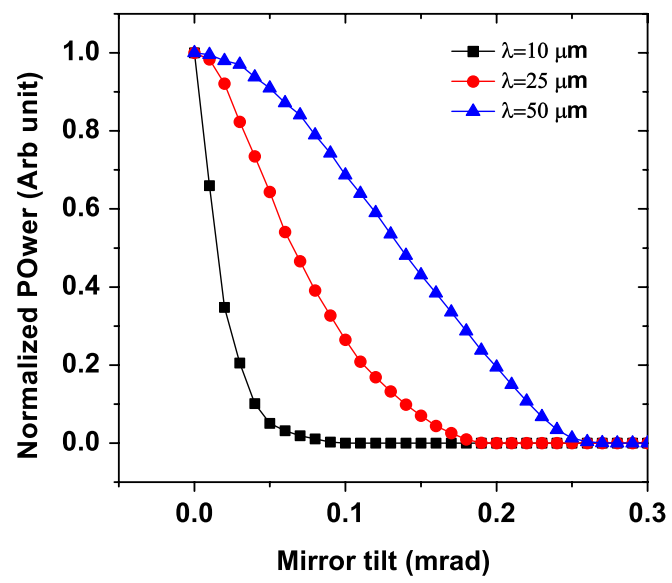

(b)

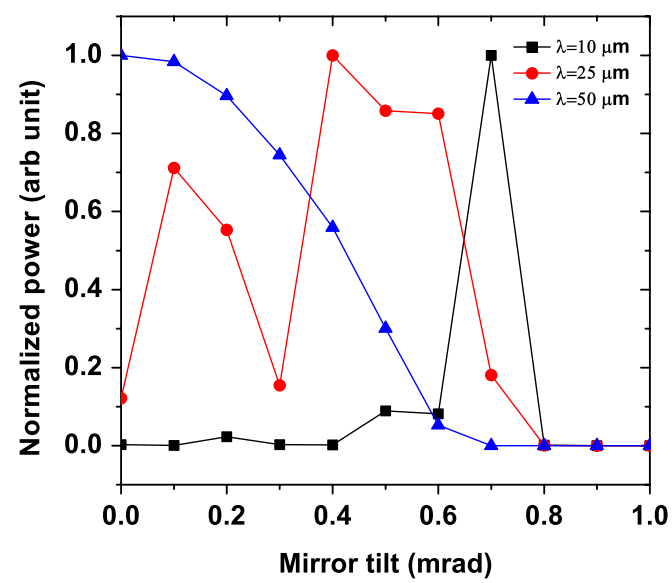

(c)

FIG. 13. Variation of outcoupled power as function of mirror tilt, for a hole size of $4 \mathrm{~mm}$, at three different wavelengths for the (a) confocal $(g=0)$, (b) concentric $(g=-1)$, and (c) intermediate $(g=-0.6)$ configurations. configurations. For the confocal configuration, Fig. 13(a), the situation is complicated. Here we find an optimal value of the mirror tilt at which the output power is greater than when there is no tilt. For a wavelength of $10 \mu \mathrm{m}$ the intensity is maximum at a tilt of $0.42 \mathrm{mrad}$, for $25 \mu \mathrm{m}$ at $0.4 \mathrm{mrad}$, and for $50 \mu \mathrm{m}$ at $0.5 \mathrm{mrad}$. This may seem surprising, suggesting that a poorly aligned mirror is better than a well aligned one in terms of outcoupled power. However, Figs. 14(a)-14(c) show that this is because with tilt the mode is completely distorted. The mode has become nonGaussian, but is also more compact. As a result, more power couples out of the hole, but this is at the cost of poor mode quality.

For the concentric configuration, Fig. 13(b), the power decreases monotonically and rapidly with increasing tilt for all wavelengths. This effect is stronger at lower wavelengths and can be explained on the basis of the modes shown in Figs. 14(d)-14(f). One can see that as the mirror tilt increases, the mode shifts off axis, while remaining approximately Gaussian. Therefore, with increasing tilt the outcoupled power just decreases, until a limit is reached beyond which there is no lasing because the reflected radiation is substantially off axis and therefore the overlap with the electron beam is poor. The mode shift is greater at shorter wavelengths, resulting in lesser outcoupled power.

For $g=-0.6$, Figs. 14(g)-14(i) show the mode profiles for a wavelength of $25 \mu \mathrm{m}$, for mirror tilts of zero, 0.1 and $0.3 \mathrm{mrad}$ respectively. Without any tilt the mode is clearly a higher-order mode, and only a modest amount of power is coupled out of the hole. With a tilt of $0.1 \mathrm{mrad}$, however,

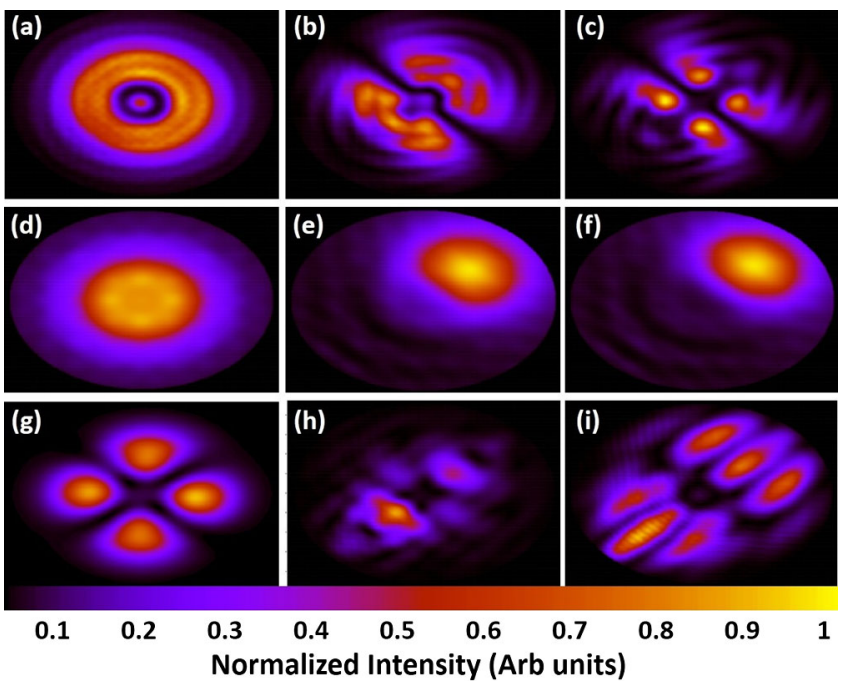

FIG. 14. Mode profiles from the simulations with a tilted mirror at a wavelength of $25 \mu \mathrm{m}$ and a hole size of $4 \mathrm{~mm}$, for the confocal configuration ( $g=0$ ) with tilts of (a) zero, (b) $0.3 \mathrm{mrad}$, and (c) $0.5 \mathrm{mrad}$; for the concentric configuration $(g=-1)$ with tilts of (d) zero, (e) $0.1 \mathrm{mrad}$, and (f) $0.3 \mathrm{mrad}$; for the intermediate configuration $(g=-0.6)$ with tilts of (g) zero, (h) $0.1 \mathrm{mrad}$, and (i) $0.3 \mathrm{mrad}$. For each plot the window size is $50 \mathrm{~mm}$ by $50 \mathrm{~mm}$. 
the mode completely changes. There is now a single intense spot, though it is somewhat displaced from the center. Therefore, there is now actually more power outcoupled through the hole. For a tilt of $0.3 \mathrm{mrad}$, the mode is again of high order, with maxima away from the hole, and hence the outcoupled power is low.

It is interesting to compare these results with experiments and simulations from the CLIO FEL [20]. CLIO has mirrors with a radius of curvature of $3 \mathrm{~m}$, and a cavity length of $4.8 \mathrm{~m}$, corresponding to $g=-0.6$, and a $2 \mathrm{~mm}$ hole in the outcoupling mirror. They find that, for this configuration, there is more outcoupled power with a mirror tilt of $0.4 \mathrm{mrad}$, compared to a perfectly aligned mirror. In their case too, they find that this is because of a change in the mode profile as a consequence of tilting the mirror.

Our simulations show that confocal configurations are more robust to mirror tilts compared to concentric configurations. In the latter, there is no lasing beyond a tilt of $0.2 \mathrm{mrad}$. This is because the longer length of the concentric resonator leads to a larger spatial displacement of the beam for a given tilt. The reflected beam therefore very quickly goes off axis, resulting in poor overlap with the electron beam which kills lasing. For the confocal and intermediate configurations, with greater values of the mirror tilt, it is possible to get more outcoupled power, but this is only because the mode is now a more compact higher-order mode. However, for the concentric configuration the mode always remains approximately Gaussian, while for the other two configurations the mode for a tilted mirror is generally substantially non-Gaussian.

\section{MODE DETERMINATION}

In many cases the observed mode is clearly nonGaussian. In principle, any cavity mode can be expanded in a basis of either Gauss-Hermite (GH) modes or GaussLaguerre (GL) modes, since each of them forms a complete basis set [11]. However, keeping in mind the cylindrical symmetry of the cavity, and the fact that we have a helical undulator, one may expect the GL to be a better basis set for the expansion, in the sense that the observed mode profile may correspond to a single higher-order GL mode. The same mode profile could, of course, be expanded in GH modes, but may require a larger number of terms in the expansion to get reasonable agreement with the observed mode.

The electric field of the laser can be expanded in the GH basis as

$$
\begin{aligned}
E_{m n}(\vec{r})= & \frac{A_{m n}}{w(z)} H_{m}\left[\frac{\sqrt{2} x}{w(z)}\right] H_{n}\left[\frac{\sqrt{2} y}{w(z)}\right] \\
& \times \exp \left[-\frac{x^{2}+y^{2}}{w^{2}(z)}\right] \exp [-i \alpha],
\end{aligned}
$$

where $H_{n}$ and $H_{m}$ are Hermite polynomials and the phase $\alpha$ is given by

$$
\alpha=K \frac{x^{2}+y^{2}}{2 R(z)}-k z-\phi_{n m}(z),
$$

where

$$
\phi_{n m}=(m+n+1) \times \tan ^{-1}\left[\frac{\lambda(z)}{\pi w^{2}(z)}\right] .
$$

Similarly, expansion in the GL basis is given by

$$
\begin{aligned}
E_{l m}(r, \phi, z)= & \frac{C_{l m}}{w(z)} e^{\left[\frac{-r^{2}}{w^{2}(z)}\right]} \times e^{-i \alpha}(-1)^{\min (l, m)} \\
& \times\left[\frac{r \sqrt{2}}{w(z)}\right]^{l-m} L_{\min (l, m)}^{l-m}\left(\frac{2 r^{2}}{w^{2}(z)}\right),
\end{aligned}
$$

where $L_{p}^{l}$ is the generalized Laguerre polynomial and the phase $\alpha$ is given by

$$
\alpha=K \frac{r^{2}}{2 R(z)}+(l+m+1) \psi+(l-m) \phi,
$$

where

$$
\psi=\tan ^{-1}\left(\frac{z}{z_{R}}\right)
$$

In order to systematically characterize these modes we wrote a MATHEMATICA [21] program that gives the $G L_{i j}$ and $G H_{i j}$ modes for given values of $i$ and $j$. The code also calculates any linear combination of GL and GH modes, which can be compared with the modes observed in the simulation to get the best agreement. Some of the modes analyzed are shown in Fig. 15, and details of the best fit GL and GH expansions are shown in Table II.

Figure 15(a) shows the mode from Fig. 9(a), where we had observed that there is no lasing. It is clear from the expansion (Table II, first row) that this is just a fourth-order GL mode. Note that the expansion in terms of GH modes requires four higher-order $\mathrm{GH}$ modes, and even then the agreement is not very good. Figure 15(b) shows another example corresponding to one of the points in Fig. 5(a), where lasing is observed. The mode looks complicated, showing many rings of different intensity, but our analysis shows that it is just a first-order GL mode (Table II, second row). Similarly, Fig. 15(c) shows a third example corresponding to another point from Fig. 5(a) where there is lasing, and again the mode is a first-order GL mode (Table II, third row). In the latter two cases the GH expansion also gives a reasonable fit, but it requires a larger combination of $\mathrm{GH}$ modes.

Table II, fourth row, shows the modal analysis for the mode shown in Fig. 9(b); the mode is complicated looking, 

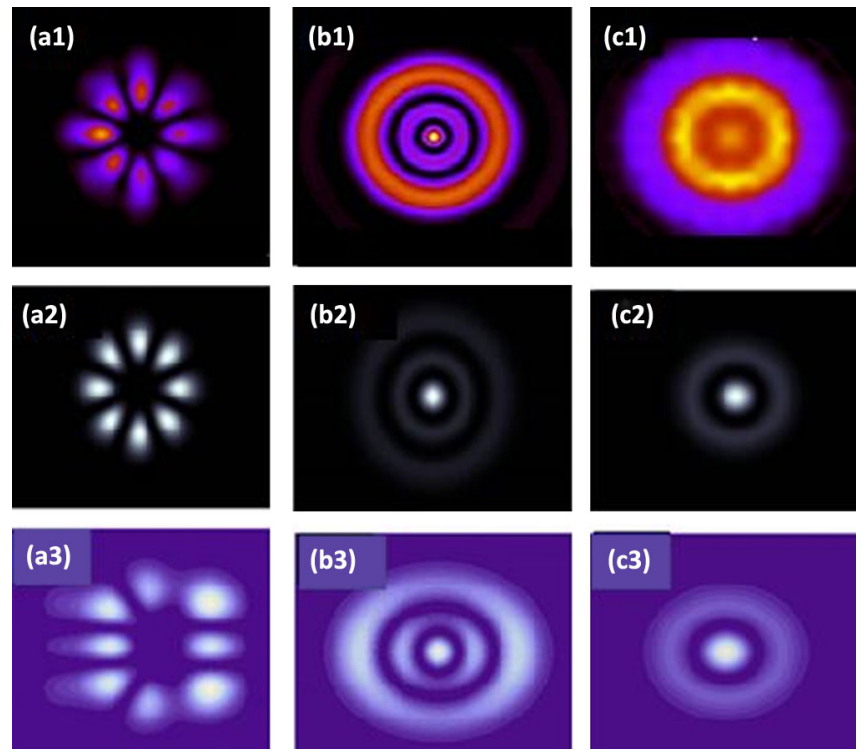

FIG. 15. Mode profiles obtained from the simulations (first row), from a Gauss-Laguerre expansion (second row), and from a Gauss-Hermite expansion (third row); (a) intermediate configuration $(g=-0.6), 10 \mu \mathrm{m}$ wavelength, $4 \mathrm{~mm}$ hole size, (b) intermediate configuration $(g=-0.9), 25 \mu \mathrm{m}$ wavelength, $1 \mathrm{~mm}$ hole, and (c) confocal configuration $(g=0), 50 \mu \mathrm{m}$ wavelength, $1 \mathrm{~mm}$ hole. Details of the mode expansions are given in Table II. For each plot the window size is $50 \mathrm{~mm}$ by $50 \mathrm{~mm}$.

but turns out to be a second order GL mode. Similarly, the last row in Table II shows that the mode for the $g=-0.6$ configuration, at a wavelength of $10 \mu \mathrm{m}$ and a hole size of $1 \mathrm{~mm}$ (for which the mode profile is not shown in this paper) is also a second order GL mode. In every case, the GL is clearly the more natural basis set for describing the modes. This is not surprising because of the axisymmetry. However, for a linear undulator things may be different.

This analysis shows that even when the dominant mode is a higher-order mode, it is generally a single GL mode, and it should therefore be possible to suppress the mode using intracavity elements, and force the dominant mode back towards the Gaussian, thus enhancing the performance of the FEL. This can be done, for example, by the use of a graded-reflectivity mirror with an intracavity phase element [22], or the inverted relief technique [23].

TABLE II. Mode profiles characterized in terms of GH and GL expansions.

\begin{tabular}{lcccc}
\hline \hline$\lambda(\mu \mathrm{m})$ & $g$ & $\begin{array}{c}\text { Hole } \\
(\mathrm{mm})\end{array}$ & $\begin{array}{c}\mathrm{GL} \\
\text { expansion }\end{array}$ & $\mathrm{GH}$ expansion \\
\hline 10 & -0.6 & 4 & $G L_{04}$ & $G H_{01}+G H_{32}+G H_{21}+G H_{10}$ \\
25 & -0.9 & 1 & $G L_{01}^{*}$ & $G H_{13}+2 G H_{00}+G H_{20}+G H_{20}$ \\
50 & 0 & 1 & $G L_{10}$ & $G H_{02}+G H_{20}$ \\
25 & -0.6 & 4 & $G L_{02}$ & $G H_{12}+2 G H_{0}+G H_{32}+G H_{20}$ \\
10 & -0.6 & 1 & $G L_{20}$ & $G H_{02}+G H_{20}+G H_{04}+G H_{22}$ \\
\hline \hline
\end{tabular}

Note that this is different from saying that any mode can be formally expanded in a GL basis; in that case there will be many GL modes, and it will not be experimentally feasible to suppress all of them. The alternate expansion, in GH modes, emphasizes this point.

\section{CONCLUSION}

We have performed a systematic simulation study of resonator stability in FEL oscillators using the codes GENESIS and OPC, varying the stability parameter $(g)$ of the cavity (through the cavity length), the wavelength of the laser, and the size of the outcoupling hole. The dependence of resonator stability on these parameters is complicated, but certain general points emerge. In order to have stable lasing over a wide range of wavelengths, the FEL cavity configuration should be carefully chosen. Broadly, the concentric configuration gives the best mode stability. The modes typically remain close to Gaussian. However, for the confocal configuration, and especially for the $g=-0.6$ intermediate configuration that we have investigated, the mode is sensitive to the wavelength and typically higher-order modes become dominant, so that the mode is no longer Gaussian and it may not be possible to lase at shorter wavelengths. This is an important observation and suggests that for FELs where wavelength tuning is an important feature, it is better to operate the resonator in the concentric configuration.

We also find that for the concentric and confocal configurations, with a larger hole size one can outcouple more power. However, intermediate configurations are extremely sensitive to the hole size, and at larger hole sizes the dominant mode can switch to a higher-order mode with an on-axis minimum, reducing the outcoupled power, and perhaps even terminating lasing.

We have also constructed a very simple matrix model of the FEL interaction and mode propagation in the cavity. It is significant to observe that for all these simulations our simple analytic model, that does not have a detailed representation of the FEL interaction, agrees extremely well with the simulations, giving the same modes. This shows that modes in FEL oscillators are determined more by the cavity configuration and radiation propagation than by the details of the FEL interaction. Therefore, our model can be used for preliminary resonator configuration studies that can serve as inputs for detailed FEL simulations.

We have also looked at the effect of mirror tilt and shown that tilting the mirror displaces the mode from the center, but can also induce higher-order modes. This can have the interesting side effect of allowing more power to be outcoupled compared to a perfectly aligned mirror, though this is at the cost of having a complicated mode structure. These simulations are in good agreement with experiments and simulations performed at the CLIO FEL.

Finally, we have also analyzed the various higher-order modes that are typically obtained, in terms of expansions in 
Gauss-Laguerre and Gauss-Hermite modes. We find that in most cases the GL is a better basis set for the expansion, and the observed dominant mode is typically a single higher-order GL mode. This is an important observation, because it suggests that it should be possible to get greater outcoupled power in such situations by the use of intracavity elements to filter out the relevant higher-order mode, leading to better FEL performance.

Our results and conclusions should be of interest and use in the design and operation of infrared FEL user facilities, where lasing over a wide wavelength range and with high outcoupled power is important.

\section{ACKNOWLEDGMENTS}

We thank Sushil Arun Samant for useful discussions, and one of us (A. P.) thanks him for help in running GENESIS. We also thank an anonymous referee for a careful reading of the manuscript and many useful comments and suggestions that have added value to the paper.

[1] J. Xie et al., Nucl. Instrum. Methods Phys. Res., Sect. A 341, 34 (1994).

[2] W. Schoellkopf et al., in Proceedings of the 34th International Free-Electron Laser Conference (FEL2012), Nara, Japan, 2012, http://jacow.org/.

[3] P. W. van Amersfoort et al., Nucl. Instrum. Methods Phys. Res., Sect. A 318, 42 (1992).

[4] T. Takii, E. Oshita, S. Okuma, K. Wakita, A. Zako, Y. Kanazawa, A. Koga, K. Ohasi, and T. Tomimasu, Nucl. Instrum. Methods Phys. Res., Sect. A 407, 21 (1998).

[5] D. C. Nguyen, R. L. Sheffield, C. M. Fortgang, J. C. Goldstein, J. M. Kinross-Wright, and N. A. Ebrahim, Nucl. Instrum. Methods Phys. Res., Sect. A 429, 125 (1999).

[6] S. Krishnagopal and A. M. Sessler, Opt. Commun. 98, 274 (1993).
[7] S. Krishnagopal, M. Xie, and K.-J. Kim, Nucl. Instrum. Methods Phys. Res., Sect. A 331, 654 (1993).

[8] S. A. Samant and S. Krishnagopal, in Proceedings of the International Particle Accelerator Conference, Kyoto, Japan (ICR, Kyoto, 2010), p. 2170.

[9] W. M. Fawley, Report Nos. LBID-2141, CBP Tech Note-104, UC-414, 1995.

[10] S. Reiche, Nucl. Instrum. Methods Phys. Res., Sect. A 429, 243 (1999).

[11] A. E. Siegmann, Lasers (University Science Books, Sausalito, California, 1986).

[12] B. Faatz, R. W. B. Best, D. Oepts, and P. W. van Amersfoort, Pure Appl. Opt. 2, 195 (1993).

[13] G. A. Barnett, S. V. Benson, and J. M. J. Madey, IEEE J. Quantum Electron. 29, 479 (1993).

[14] MATLAB and Statistics Toolbox Release 2012b, The MathWorks, Inc., Natick, Massachusetts, United States.

[15] H. P. Freund and T. M. Antonsen, Jr., Principles of FreeElectron Lasers, 2nd ed. (Chapman and Hall, London, 1996).

[16] OPC design team, http://lpno.tnw.utwente.nl/index.php? mod=research\&projectid $=21 \&$ contentid $=11$.

[17] B. Faatz, R. W. B. Best, D. Oepts, and P. W. van Amersfoort, Nucl. Instrum. Methods Phys. Res., Sect. A 331, 636 (1993).

[18] B. Faatz, R. W. B. Best, D. Oepts, and P. W. van Amersfoort, IEEE J. Quantum Electron. 29, 2229 (1993).

[19] R. Prazeres, J. M. Berset, R. Chaput, F. Glotin, D. A. Jaroszynski, and J. M. Ortega, Nucl. Instrum. Methods Phys. Res., Sect. B 89, 54 (1994).

[20] R. Prazeres, J.-P. Berthet, F. Glotin, J.-M. Ortega, B. Biswas, and U. Kale, Phys. Rev. ST Accel. Beams 13, 090702 (2010).

[21] Wolfram Research, Inc., MathematicA, Version 9.0, Champaign, IL, 2012.

[22] S. Makki and J. Leger, IEEE J. Quantum Electron. 37, 80 (2001).

[23] R. P. Sarzaa and W. Nakwaski, Photon. Lett. Poland 6, 29 (2014). 